From the Departments of Pathology and Medical Oncology/Hematology, the Netherlands Cancer Institute; Agendia BV; Department of Hematology, Academic Medical Center, Amsterdam, the Netherlands; Department of Pathology, Central Laboratories, Friesland; Department of Pathology, Radboud University Medical Center Nijmegen; Department of Hematology, Radboud University Medical Center Nijmegen, Nijmegen; Department of Pathology, University Medical Center Groningen, Groningen, the Netherlands; and the Experimental Medicine Unit, Université Catholique de Louvain, Belgium.

Submitted February 18, 2006; accepted November 8, 2006; published online ahead of print at www.jco.org on January 2, 2007

Supported by a grant from the Belgian Federation Against Cancer (L.K.)

A.M.G. and L.K. contributed equally to this study

Authors' disclosures of potential conflicts of interest and author contributions are found at the end of this article.

Address reprint requests to Daphne de Jong, Department of Pathology, The Netherlands Cancer Institute, Plesmanlaan 121, 1066 CX Amsterdam, the Netherlands; e-mail: d.d.jong@nki.nl.

() 2007 by American Society of Clinical Oncology

0732-183X/07/2504-390/\$20.00

DOI: $10.1200 / J C O .2006 .06 .1648$

\title{
Gene-Expression and Immunohistochemical Study of Specific T-Cell Subsets and Accessory Cell Types in the Transformation and Prognosis of Follicular Lymphoma
}

Annuska M. Glas, Laurent Knoops, Leonie Delahaye, Marie José Kersten, Robby E. Kibbelaar, Lodewyk A. Wessels, Ryan van Laar, J. Han J.M. van Krieken, Joke W. Baars, John Raemaekers, Philip M. Kluin, Laura J. van 't Veer, and Daphne de Jong

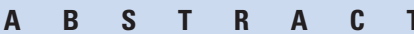

\section{Purpose}

Despite the generally favorable clinical course in follicular lymphoma (FL), a minority of patients have a poor prognosis-with death within 3 years of diagnosis-most often due to transformation to aggressive disease.

\section{Patients and Methods}

In this study, we analyzed the potential of predicting early transformation on the basis of gene expression and immunologic parameters in FL biopsy samples taken at diagnosis.

\section{Results}

At the gene-expression level, FL is a highly uniform disease at the time of diagnosis, precluding the detection of sufficiently validated prognostic gene-expression profiles suitable for a clinical setting. Combinations of differentially expressed genes indicate that immunologic mechanisms play a differential role in the risk of early transformation. Using immunohistochemistry for specific cell populations, the spatial distribution to neoplastic follicles and the activation of CD4-positive T-helper cells $(P=.002)$ and specifically T-helper $1(P=.004)$ were shown to be highly discriminatory to predict early transformation. A role for functional modulation of follicular dendritic cells could also be supported $(P=.04)$. Other cell populations, including CD68-positive macrophages and regulatory $T$ cells, were not differentially present.

\section{Conclusion}

These results support the identification of FL as an immunologically functional disease in which an interaction of the tumor cells and the functional composition of the microenvironment determines the clinical behavior

\section{J Clin Oncol 25:390-398. (c) 2007 by American Society of Clinical Oncology}

\section{INTRODUCTION}

Follicular lymphoma (FL) is the most frequent indolent B-cell lymphoma in adults. Generally, the disease responds well to chemotherapy, but it is characterized by frequent relapses. Despite many different treatment approaches, survival has not improved over the past decades. The disease is still considered to be incurable. Transformation to aggressive disease in the form of diffuse large B-cell lymphoma (DLBCL) or to a lesser extent the development of nonresponsiveness to therapy without transformation form the major causes of diseaserelated death. ${ }^{1,2}$

Although a long, indolent course of the disease is considered to be prototypical, the variation in survival is quite broad with up to $15 \%$ of the patients dying early in the course of their disease. A combined score of clinical parameters, such as the Follicular Lymphoma International Prognostic Index, is considered the most successful prognosticator thus far, but does not reveal any biologic mechanisms that play a role in transformation and prognosis. ${ }^{3}$ Furthermore, to date very few biologic prognosticators with clinical utility have been identified. Histologic grading does correlate with clinical outcome both for disease-free and overall survival. ${ }^{4}$ Its usefulness for individual patient management is hampered, however, by its subjective nature and poor reproducibility. ${ }^{5}$ Most consistently, a complex karyotype is indicative of an adverse prognosis. ${ }^{6,7}$ This is in line with the current model of transformation being the result of an accumulation of genetic alterations and possibly an inadequate repair process. ${ }^{8}$ 
Previous immunohistochemical studies, and more recently gene-expression studies, have lent support to a model of FL as a disease of immunologically functional cells in which an interaction of the tumor cells and the microenvironment determines the clinical behavior. $^{9-13}$ It has been hypothesized that the specific genomic alterations in FL cells may actually dictate the functional composition of the microenvironment. ${ }^{8}$ The cellular composition of the nonmalignant infiltrate that is related to prognosis and the mechanisms that play a role are not elucidated yet.

In this study, we explored models on pathogenesis in FL using gene-expression profiling and immunohistochemistry concentrating on the risk of transformation as an objective and disease-related biologic end point.

\section{PATIENTS AND METHODS}

\section{Patients}

From the files of the departments of pathology of the contributing institutions, three series of samples from patients with FL were collected for gene-expression studies: representative frozen tissue of biopsy samples of 31 patients who showed histologically or cytologically proven transformation to DLBCL within 3 years after diagnosis; representative frozen tissue of samples of 35 patients with fully documented minimum follow-up of 7 years and without clinical, histologic, or cytologic signs of transformation; and representative frozen tissue of biopsy samples of 24 patients with DLBCL as transformation of previously diagnosed FL.

Biopsy samples of FL were included when taken before treatment or during follow-up after a varying time of watch and wait. Samples were included as well at time of relapse if biopsy site was outside the field of radiotherapy after local radiotherapy as initial treatment. Time of diagnosis ranged from 1986 to 2004 and treatment protocols were various, including chlorambucil, combination chemotherapy (cyclophosphamide, vincristin, prednisone), and radiation therapy. Patients with FL treated with high-dose chemotherapy and autologous peripheral stem-cell or bone marrow transplantation outside the context of transformed disease were excluded from the study. None of the patients received rituximab.

For immunohistochemical analysis, representative formalin-fixed and paraffin-embedded biopsy samples from 25 patients with transformation within 3 years using the same selection criteria as discussed earlier were collected. Of these, 15 were also included in the gene-expression study. Thirtythree instances of patients without signs of transformation during a minimum follow-up time of 7 years (as described) were included, 15 of which were also included in the gene-expression analysis. Clinical characteristics of all patients are listed in Table 1.

\section{Gene-Expression Analysis}

RNA isolation, amplification, labeling, and hybridization were performed as previously described. ${ }^{9}$ Detailed protocols can be found on the NKI/AvL Department of Pathology Microarray Projects Web page. ${ }^{14}$ All samples were cohybridized with a standard reference of pooled and amplified RNA isolated from five routine tonsillectomy specimens, chosen and optimized to identify small changes in expression levels between the tumor groups of interest.

cDNA microarray slides, with a complexity of 19,200 spots per slide, were prepared at the Central Microarray Facility at the Netherlands Cancer Institute. ${ }^{15}$

The description of this study follows the minimum information about a microarray experiment (MIAMI) guidelines issued by the Microarray Gene Expression Data group. ${ }^{16}$

\section{Immunohistochemistry}

Immunohistochemical stainings were performed on complete paraffin sections of FL samples according to standard antigen microwave/citrate-based retrieval techniques. Antibodies used are listed in Table 2.
For CD20, the pattern of interfollicular tumor cell distribution was scored. MIB1 staining was assessed semiquantitatively as a proportion of total number of tumor cells. T-cell subpopulations were semiquantitatively assessed in a four-tiered fashion $(0 \%$ to $1 \% ; 1 \%$ to $5 \% ; 5 \%$ to $10 \%$; > $10 \%$ ) relative to total numbers of T-cells and spatial distribution was scored as predominantly intrafollicular, predominantly interfollicular, or diffuse. CD69 as a marker for $\mathrm{T}$-cell activation was scored relative to the total number of $\mathrm{T}$ cells based on pattern recognition of the infiltrate. CD69 positivity on tumor cells was not taken into account. CD21 and CD23 as markers for follicular dendritic meshworks were scored in a four-tiered system (absent, minority of the neoplastic follicles with disrupted meshworks, majority of neoplastic follicles with welldeveloped meshworks, uniformly well-developed meshworks). CD68positive macrophages were counted as absolute cell numbers per three representative follicular high-power fields (at magnification $\times 600$ ). All scorings were performed in a blinded fashion for clinical outcome.

\section{Statistical Analysis}

Both for the gene-expression study and the immunohistochemical analysis, we chose to compare the instances of clinical spectrum extremes, rather than random samples of FL reflecting an unbiased clinical distribution. ${ }^{9,17}$ Because the far majority of FL has a favorable prognosis and only approximately $20 \%$ of the patients showed early transformation and/or a poor prognosis, an unbiased series would show a significant under-representation of poor prognosis patients. This would potentially inhibit the recognition of minor but biologically significant information in this group. The presently used choice, however, precludes formal survival and Cox regression analysis. As a consequence of the protracted clinical course in the majority of the patients and the dominant elderly age group, patients often die of other causes than of lymphoma. Therefore, we concentrated on disease-related survival rather than overall survival.

\section{Unsupervised Analysis}

Unsupervised analysis was performed on a selection of the total patient series consisting of 24 patients with early proven transformation; 22 patients without transformation; and 24 patients with DLBCL as transformation of previously diagnosed FL.

Gene clustering was performed on 9,441 significantly differentially expressed genes, based on the Rosetta error model. ${ }^{18}$ Agglomerative hierarchical clustering was performed using complete linkage similarity metrics in Genesis. ${ }^{19}$

Supervised analysis of the FL gene-expression data was approached using several computational strategies based on the Shrunken Centroids method in the significance analysis of microarrays (SAM) software on a selection of 24 patients with rapid transformation to DLBCL and 22 patients without transformation ${ }^{20}$ and on signal-to-noise ratios on a similar selection of patients groups.

For construction of a formal predictive gene-expression profile, genes that optimally separated the two classes best were determined by using different classifier methods in a train and validation protocol as described by Wessels et al. ${ }^{21,22}$

\section{RESULTS}

\section{Unsupervised Analysis Can Distinguish FL From DLBCL}

As a first step, unsupervised two-dimensional hierarchical clustering was performed on $46 \mathrm{FL}$ samples including both clinical groups and 24 DLBCL samples. This showed a clear separation between FL and DLBCL, with one cluster containing 75\% of DLBCL samples (18 of 24) and only 7\% of FL samples (three of 45; Fig 1). Unsupervised clustering was not able to distinguish clinically favorable FL $(\mathrm{n}=22)$ and unfavorable FL $(\mathrm{n}=24)$, underlining the biologic homogeneity of this disease. 
Table 1. Clinical Characteristics of the Patients in the Gene Expression and Immunohistochemical Studies

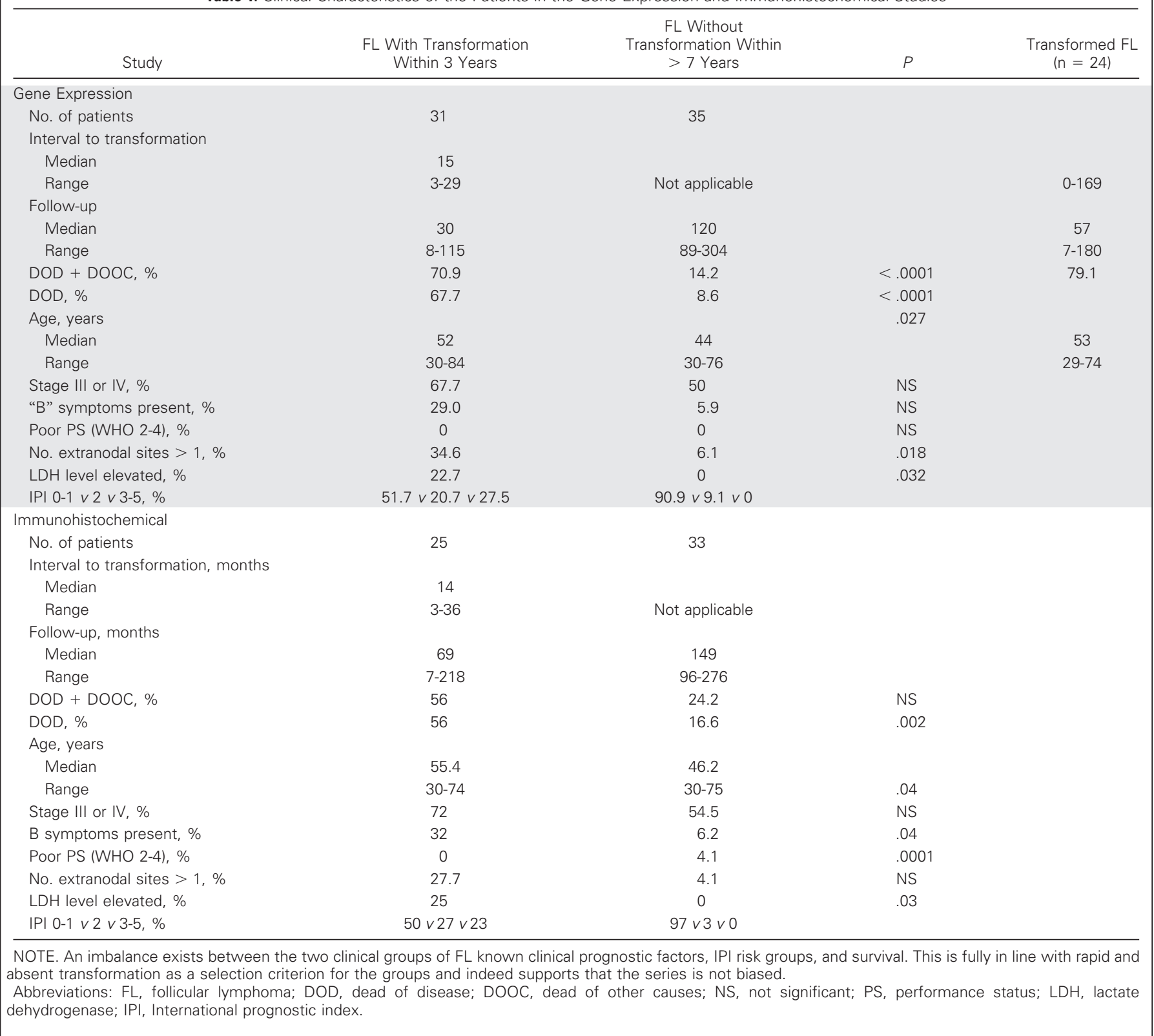

\section{Discriminatively Expressed Genes Between Clinically Distinct Groups of FL Are Comparable to Genes Expressed in Activated Lymphoid Tissue}

To focus on gene-expression differences between both clinical groups, reporter genes were selected using SAM as well as signalto-noise ratios.

SAM analysis of nontransforming FL ( $\mathrm{n}=22$ patients) versus rapidly transforming FL $(n=24)$ resulted in 86 genes with significantly different expression between the two groups (with a false discovery rate of $8 \%$ ). Signal-to-noise ratios (SNRs) were calculated for a selection of patients representing the clinical extremes of the spectrum (Fig. 2).

Gene ontology analysis revealed that both the classical SNR approach and the SAM approach showed a significant over- representation between both clinical FL groups of the genes involved in immunologic functions (30\% overall of SAM and SNR identified genes). Detailed information on these genes and functional grouping is listed in Table 3. The majority of these immune response-related genes were expressed at relatively higher levels in rapidly transforming FL than in nontransforming FL (25 of 30 markers, excluding the various immunoglobulins). Moreover, the relative expression levels in the rapidly transforming FL were very similar or relatively increased to normal lymphoid tissue with follicular hyperplasia, which was used as a hybridization reference, while their expression was relatively downregulated in nontransforming FL. Therefore, the rapidly transforming group showed an overall higher similarity of gene expression to follicular hyperplasia. 


\begin{tabular}{|ccl}
\hline \multicolumn{3}{c}{ Table 2. Antibodies Used in This Study } \\
\hline Antibody & Clone & \multicolumn{1}{c}{ Source and Location } \\
\hline Ki-67 & MIB1 & DAKO, Glostrup, Denmark \\
CD20 & L26 & DAKO \\
CD3 & CD3 & DAKO \\
CD4 & 4B12 & Novacastra, Newcastle Upon Tyne, United Kingdom \\
CD8 & C8/144B & DAKO \\
T-bet & anti-T-bet & Zymed, Invitrogen, Breda, the Netherlands \\
CD57 & leu-7 & Becton Dickinson, Franklin Lakes, NJ \\
CD56 & 123C3.D5 & NeoMarkers \\
FOXP3 & 236 A/E7 & AbCam, Cambridge, United Kingdom \\
CD21 & 1F8 & DAKO \\
CD23 & 1B12 & Novacastra \\
CD68 & KP1 & DAKO \\
CD69 & CH11 & Lab Vision, Fremont, CA \\
\hline
\end{tabular}

Abbreviation: FOXP3, forkhead box protein P3.

\section{Translation of Gene-Expression Patterns to Lymphoid Cell Populations Using Immunohistochemistry}

To further define the cell types involved in prognosis in FL and to analyze the variations in relative cell numbers and the activation state of specific cell populations, immunohistochemical studies were per- formed. The results are summarized in Table 4 and illustrated in Figure 3. There were no significant differences in tumor cell distribution and total numbers of T cells. Most importantly, in FL with rapid transformation, CD4-positive $\mathrm{T}$ cells were predominantly found within the neoplastic follicles with or without an interfollicular component. In contrast, the CD4-positive $\mathrm{T}$ cells were predominantly found between the neoplastic follicles in the nontransforming patients. No differences were found for CD8- and CD57-positive populations. Virtually no CD56 positive natural killer cells were seen in FL samples. Specific cell populations of T-helper1 cells and regulatory $\mathrm{T}$ cells were studied using T-bet and forkhead box protein P3 (FOXP3) immunostainings, respectively. T-helper1 cells were present as a minor interfollicular population with significantly higher densities in samples from the rapidly transforming patients, but never exceeding $1 \%$ of the total T-cell numbers. Regulatory $\mathrm{T}$ cells were present at varying frequencies in approximately half of the cases containing less than $5 \%$ positive cells per total number of T cells ( 26 of 53 ). Cases with distinctly denser infiltrates of FOXP3-positive cells were not significantly different between the two clinical groups.

The activation state of the $\mathrm{T}$ cells was significantly higher $(P=.017)$ in the rapidly transforming patients with uniform expression of CD69 as an activation marker. In three patients, tumor B cells uniformly expressed CD69. In all remaining patients,

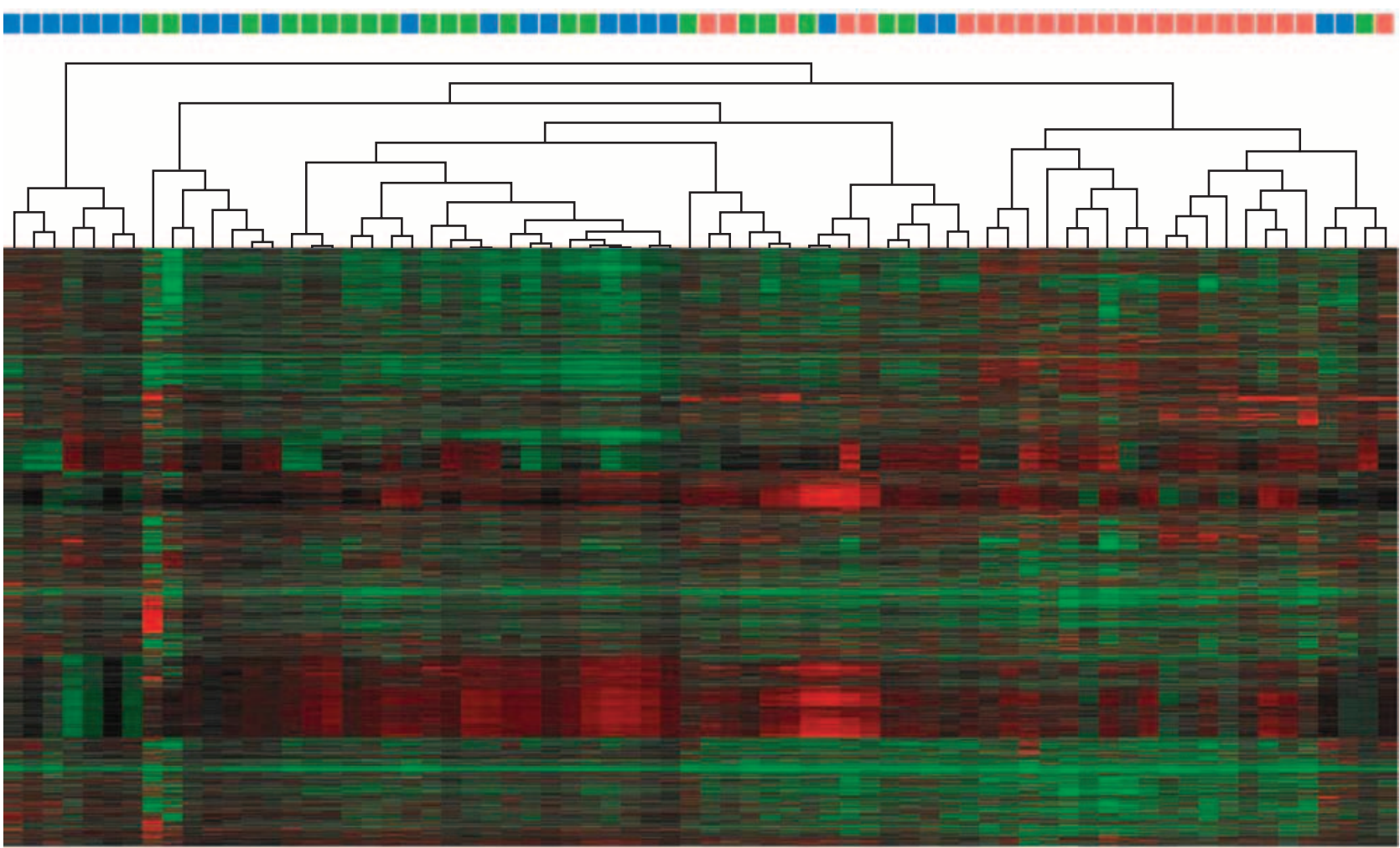

FL without transformation, minimum follow-up 7 years

FL with transformation within 3 years after diagnosis

DLBCL as transformation of FL

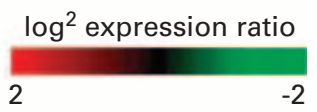

Fig 1. Unsupervised two-dimensional hierarchical clustering on 45 follicular lymphoma (FL) samples and 24 diffuse large B-cell lymphomas (DLBCL). FL and DLBCL

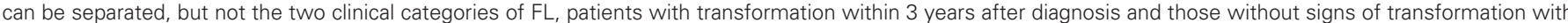
a follow-up of at least 7 years. 


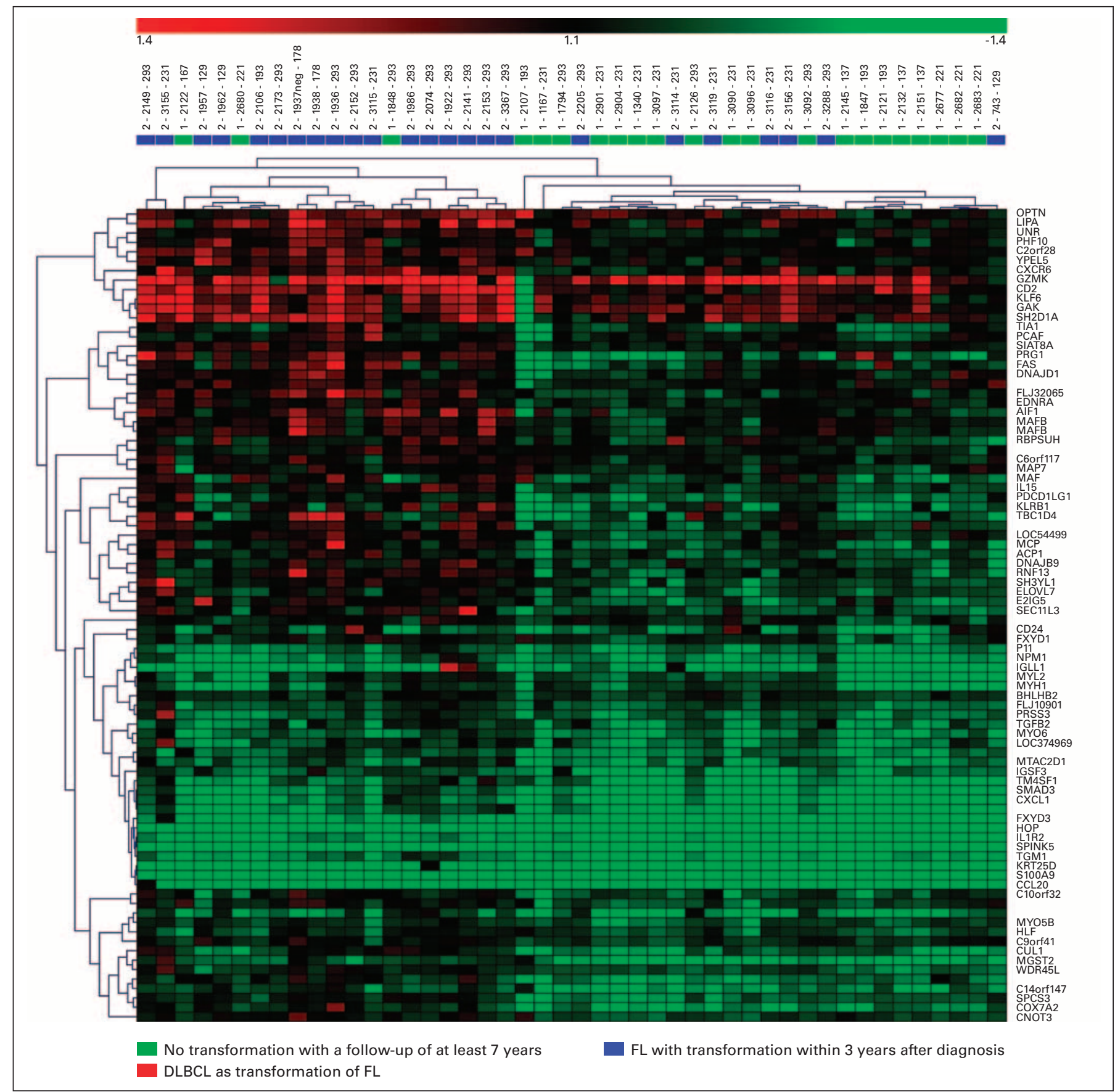

Fig 2. Clustering based on 86 genes that were significantly different in gene expression between both clinical groups of follicular lymphoma (FL) according to significance analysis of microarrays (SAM) analysis is able to separate both groups with a $78 \%$ correct stratification. However, because leave-one-out cross validation and independent validation do not make up part of the SAM analysis, this should not be considered as a predictor profile. DLBCL, large B-cell lymphoma.

the architectural pattern of CD69-positive cells matched the spatial distribution of T cells.

CD21 stainings showed that more frequently a disrupted or absent immunohistochemical pattern in the majority of neoplastic follicles of the rapidly transforming patients $(P=.04)$. Because $\mathrm{CD} 23$ was not differentially expressed, this may reflect a functional modulation rather than a physical absence of follicular dendritic cells.

CD68-positive cells showed an equal spatial distribution in both groups. No differences in mean absolute numbers nor in the proportion of patients with tissue macrophages exceeding the up-

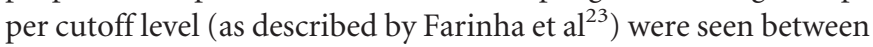
the two groups.

\section{Differences in Gene Expression Are Heterogeneous and Minor, Precluding the Construction of a Reliable Profile That Can Predict Transformation}

To evaluate whether future transformation can be predicted based on gene expression, 10 different commonly used classification 
Table 3. Differentially Expressed Immune-Related Genes Between Rapidly Transforming and Nontransforming FL by SAM Analysis and Classical SNR Related to Functional Cell Populations

\begin{tabular}{|c|c|c|c|}
\hline Function/Gene & $\begin{array}{l}\text { SNR/SAM } \\
\text { Analysis }\end{array}$ & $\begin{array}{c}\text { Expression Nearest to } \\
\text { Reference Follicular } \\
\text { Hyperplasia }\end{array}$ & $\begin{array}{c}\text { Relative Expression } \\
\text { in Rapidly } \\
\text { Transforming FL to } \\
\text { Nontransforming } \\
\text { FL }\end{array}$ \\
\hline \multicolumn{4}{|c|}{$\begin{array}{l}\text { B-cell differentiation related } \\
\text { genes }\end{array}$} \\
\hline$P B X 1$ & Both & Rapidly transforming FL & Increased \\
\hline Immunoglobulins & Both & Various & Various \\
\hline CD138 & SNR & Nontransforming FL & Increased \\
\hline CD9 & SNR & Rapidly transforming FL & Increased \\
\hline$L Y N$ & SNR & Nontransforming FL & Decreased \\
\hline \multicolumn{4}{|l|}{$\begin{array}{l}\text { T-cell subset and function } \\
\text { related genes }\end{array}$} \\
\hline CD69 & SAM & Rapidly transforming FL & Increased \\
\hline \multicolumn{4}{|l|}{$\begin{array}{l}\text { Differentiation and } \\
\text { activation }\end{array}$} \\
\hline CD101 & Both & Rapidly transforming FL & Increased \\
\hline MAL2 & SNR & Rapidly transforming FL & Increased \\
\hline$C D 2$ & SAM & Nontransforming FL & Increased \\
\hline CXCL1 & SAM & Rapidly transforming FL & Increased \\
\hline$I L-1 R$ & Both & Rapidly transforming FL & Increased \\
\hline PTPRC & SNR & Nontransforming FL & Decreased \\
\hline PTPRB & SNR & Rapidly transforming FL & Decreased \\
\hline PTPRF & SNR & Rapidly transforming FL & Decreased \\
\hline \multicolumn{4}{|l|}{ Natural killer/T function } \\
\hline LIPA & SAM & Nontransforming FL & Increased \\
\hline TIA1 & SAM & Nontransforming FL & Increased \\
\hline GZM-K & SAM & Nontransforming FL & Increased \\
\hline$K L R B 1$ & SAM & Rapidly transforming FL & Increased \\
\hline SH2D1A & SAM & Rapidly transforming FL & Increased \\
\hline CXCR6 & SAM & Nontransforming FL & Increased \\
\hline SLP1 & SNR & Rapidly transforming FL & Increased \\
\hline TM4SF1 & Both & Rapidly transforming FL & Increased \\
\hline$R A B 27 B$ & SAM & Rapidly transforming FL & Increased \\
\hline \multicolumn{4}{|l|}{ T helper1 function } \\
\hline STAT4 & SAM & Rapidly transforming FL & Increased \\
\hline CCL2O & Both & Rapidly transforming FL & Increased \\
\hline ELF3 & SNR & Rapidly transforming FL & Increased \\
\hline IFN- $\gamma$ IP16 & SNR & Nontransforming FL & Decreased \\
\hline IFN-induced TMP3 & SNR & Rapidly transforming FL & Increased \\
\hline \multicolumn{4}{|l|}{ Accessory cell function } \\
\hline CXCL1 & Both & Rapidly transforming FL & Increased \\
\hline CCL19 & SNR & Nontransforming FL & Increased \\
\hline CDC42BPK & SNR & Nontransforming FL & Increased \\
\hline
\end{tabular}

NOTE. Relative gene-expression levels in both clinical groups are compared with follicular hyperplasia that was used as a reference and the clinical groups were compared with each other. An overall higher expression of immunerelated genes in the rapidly transforming group and relative similarity or even increased expression to the expression levels in follicular hyperplasia is seen. Abbreviations: SAM, significance analysis of microarrays; SNR, signal-tonoise ratio; FL, follicular lymphoma; IL, interleukin; IFN, interferon.

methods were explored as previously published (Wessels et $\mathrm{al}^{21}$ ). Performance data for each classifier, as well as the median number of selected genes for the classifier, is given in Table 5 and show that none of the methods resulted in a validated performance exceeding $55.8 \%$.

Therefore, despite distinct biologic differences at the cell populations level between both groups, these differences at the gene-expression level are minor and of a heterogeneous nature, preventing the construction of a reliable, diagnostic geneexpression profile that may be useful in clinical practice. Because the essential biologic differences are found at the level of cell populations, immunohistochemistry is the more sensitive and informative technique to study this issue rather than gene-expression profiling. A similar classifying approach was taken to evaluate the

\begin{tabular}{|c|c|c|c|}
\hline Parameter & $\begin{array}{c}\text { FL With } \\
\text { Transformation } \\
\text { Within } 3 \text { Years }\end{array}$ & $\begin{array}{c}\text { FL Without } \\
\text { Transformation Within } \\
>7 \text { Years }\end{array}$ & $P$ \\
\hline \multicolumn{4}{|l|}{ Grade } \\
\hline За & $1 / 25$ & 2/33 & $N S^{*}$ \\
\hline \multicolumn{4}{|l|}{ Ki67 } \\
\hline$>10 \%$ of tumor cells & $7 / 24$ & $4 / 33$ & $N S^{*}$ \\
\hline \multicolumn{4}{|l|}{ CD20 } \\
\hline Interfollicular component & $11 / 24$ & $10 / 29$ & $N S^{*}$ \\
\hline \multicolumn{4}{|l|}{ CD3 } \\
\hline Dense infiltrate & $9 / 25$ & 9/33 & $\mathrm{NS}^{*}$ \\
\hline \multicolumn{4}{|l|}{ CD4 } \\
\hline Predominantly intrafollicular & $10 / 24$ & $2 / 32$ & $.0023^{*}$ \\
\hline Predominantly interfollicular & $3 / 24$ & $20 / 32$ & $.0003^{*}$ \\
\hline \multicolumn{4}{|l|}{ CD8 } \\
\hline$>10 \%$ of $\mathrm{T}$ cells & $11 / 25$ & $8 / 33$ & $\mathrm{NS}^{*}$ \\
\hline \multicolumn{4}{|l|}{ T-bet } \\
\hline$>0.5 \%$ of $\mathrm{T}$ cells & $10 / 24$ & $1 / 33$ & $.004^{*}$ \\
\hline \multicolumn{4}{|l|}{ CD57 } \\
\hline$>10 \%$ of $\mathrm{T}$ cells & $4 / 16$ & $3 / 23$ & $N S^{*}$ \\
\hline \multicolumn{4}{|l|}{ FOXP3 } \\
\hline$>10 \%$ of $\mathrm{T}$ cells & $7 / 21$ & $4 / 32$ & $\mathrm{NS}^{*}$ \\
\hline \multicolumn{4}{|l|}{ FOXP3 } \\
\hline Predominantly interfollicular & $10 / 21$ & $22 / 32$ & $\mathrm{NS}^{*}$ \\
\hline \multicolumn{4}{|l|}{ CD21 } \\
\hline Disrupted meshworks & $10 / 25$ & $5 / 33$ & $0.040^{*}$ \\
\hline \multicolumn{4}{|l|}{ CD23 } \\
\hline Disrupted meshworks & $7 / 21$ & $16 / 32$ & $\mathrm{NS}^{*}$ \\
\hline \multicolumn{4}{|l|}{ CD68 } \\
\hline Median per $3 \mathrm{HPF}$ & 32 & 34 & \\
\hline Range & $15-77$ & $19-80$ & NSt \\
\hline \multicolumn{4}{|l|}{ CD69 } \\
\hline$>50 \%$ of the T cells & $18 / 24$ & $14 / 33$ & $.017^{*}$ \\
\hline \multicolumn{4}{|c|}{$\begin{array}{l}\text { NOTE. Using the same classifier approaches to construct a formal classifying } \\
\text { profile on the basis of immunohistochemical data as described for the } \\
\text { gene-expression data, all methods yielded a classifier consisting of CD4, } \\
\text { CD21, CD69, and T-bet with significant classification ability }(P<.01) \text { and an } \\
\text { accuracy of } 61 \% \text { to } 70 \% \text {. } \\
\text { Abbreviations: FL, follicular lymphoma; NS, not significant; FOXP3, forkhead } \\
\text { box protein P3; HPF, high-power field. } \\
\text { "Fisher's exact test. } \\
\text { †Mann-Whitney U test. }\end{array}$} \\
\hline
\end{tabular}

immunohistochemical data (Table 4) and yielded a classifier consisting of the four significantly different markers.

\section{DISCUSSION}

This gene-expression study shows that FL is at the molecular level a highly uniform disease at the time of diagnosis. The differences at the gene-expression level between the extremes of the clinical spectrum are minor and heterogeneous despite major clinical differences in terms of risk of transformation and prognosis. These minor differences at the gene-expression level do contribute to important biologic insights, however. More than $60 \%$ of the most discriminative genes between FL with documented transformation to DLBCL and FL that did not transform within at least 7 years after diagnosis are involved in the cellular immune response and inflammatory processes. Globally, gene-expression patterns in rapidly transforming FL are more comparable to those in nonmalignant follicular hyperplasia, 


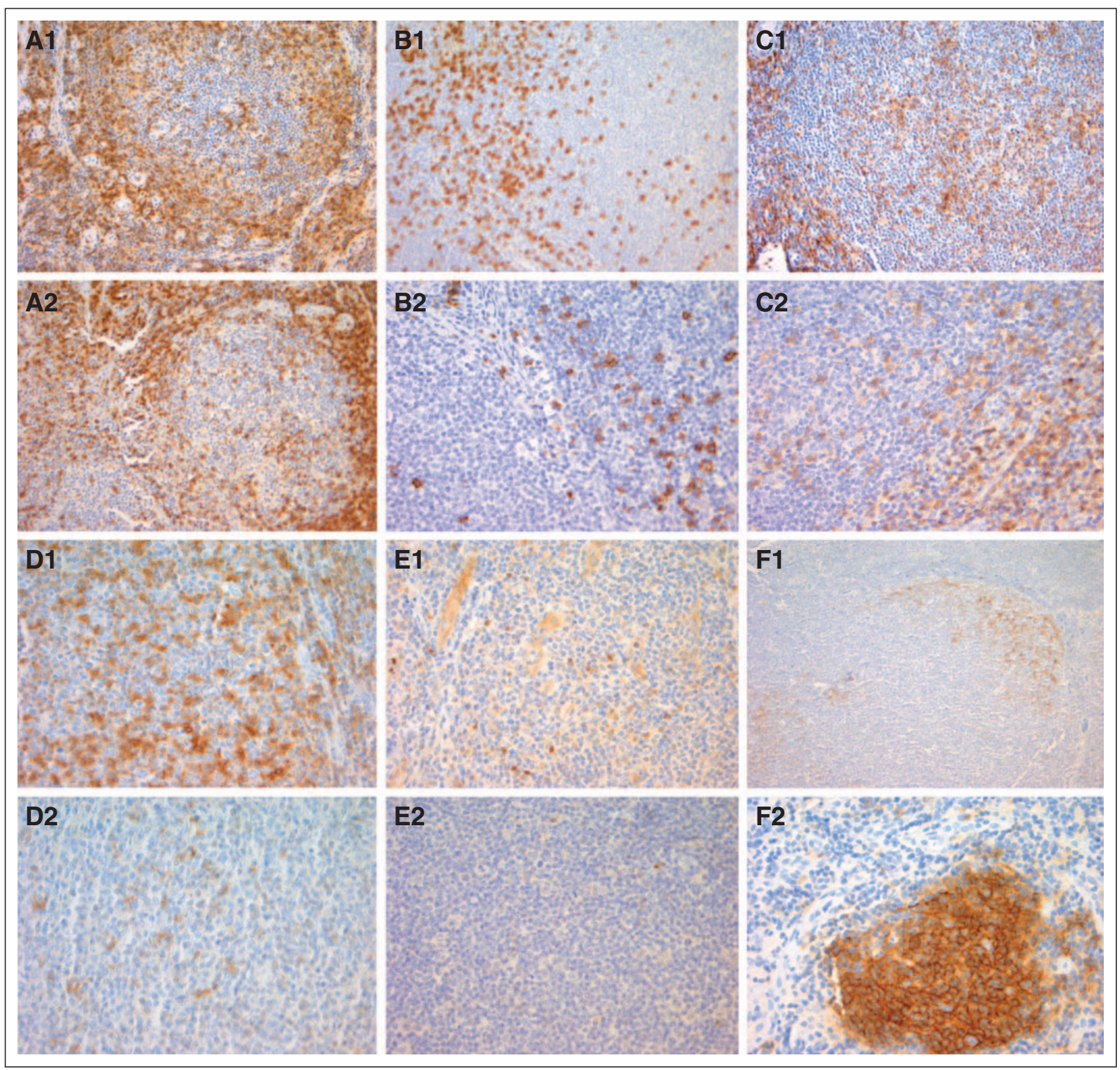

Fig 3. Immunohistochemical stainings on full sections of biopsy samples of rapidly transforming follicular lymphoma (FL; panel 1) and nontransforming $F L$ (panel 2) show different densities and spatial distributions between both clinical groups. (A) CD3; (B) CD8; (C) CD4; (D) CD69; (E) T-bet; (F) CD21.

while nontransforming FL shows overall downregulation of immune-related genes and therefore may rather resemble nonactivated lymphoid tissue.

Bohen et $\mathrm{al}^{12}$ found similar differences when studying geneexpression profiles of FL at diagnosis in relation to response to rituximab therapy that was given more than 5 years later during the course of disease. It was shown that nonresponding FL displays a geneexpression pattern that is more similar to nonmalignant lymphoid tissue (hyperplastic tonsil). This indicates that both rapidly transforming FL and rituximab nonresponding FL are characterized by an active immune response. This may be directly involved in the specific bio- logic characteristics and growth requirements of these FL groups, possibly as a stimulatory microenvironment.

Dave et $\mathrm{al}^{10}$ described gene-expression patterns in FL in relation to prognosis, showing a predominance of T-cell-related genes in good-prognosis FL and expression of accessory cell genes associated with poor prognosis. At the individual gene level, however, there is limited overlap with our genes found to be related to transformation and transformation-related prognosis. Because a custom-made statistical approach with a relative weighing of sets of genes was used by these authors, complete comparison and cross validation on independent series is limited. No obvious separation of transformed versus 


\begin{tabular}{|c|c|c|}
\hline \multicolumn{3}{|c|}{$\begin{array}{l}\text { Table 5. Validation Performance of Different Predictive } \\
\text { Gene-Expression Profiles }\end{array}$} \\
\hline Variable & $\begin{array}{l}\text { Mean Validation } \\
\text { Performance (\%) }\end{array}$ & $\begin{array}{c}\text { Median No. } \\
\text { of Selected } \\
\text { Genes }\end{array}$ \\
\hline Nearest mean & 46.5 & 6 \\
\hline Diagonal linear discriminant & 49.4 & 1 \\
\hline Simple Bayes Gaussian & 49.1 & 36 \\
\hline \multicolumn{3}{|l|}{ Nearest neighbor } \\
\hline 1 & 57 & 259 \\
\hline 5 & 47 & 101 \\
\hline 9 & 50 & 11 \\
\hline \multicolumn{3}{|c|}{ Regularized fisher linear discriminant } \\
\hline 0 & 55.8 & 21 \\
\hline 1 & 50.1 & 281 \\
\hline 10 & 47.7 & 1 \\
\hline PAM & 46 & 66 \\
\hline
\end{tabular}

nontransforming FL could be achieved using the gene selection as reported by this group (data not shown). It should be noted that the patient selection (ends-of-spectrum $v$ continuous spectrum) and end points (transformation $v$ overall survival) are essentially different between Dave et $\mathrm{al}^{10}$ and our study, precluding strong conclusions based on such comparisons.

Combinations of differentially expressed genes identified by this study point at a role for different T-cell populations, as well as accessory cell populations, such as follicular dendritic cells in FL transformation. Such differences can be more readily studied at the cellular level using immunohistochemistry. Using this approach, the activation of the immune reaction was demonstrated by the observation of increased expression of the activation marker CD69 on T cells. Few patients showed uniform expression of CD69 in tumor cells without clinical correlations. CD4-positive T-helper cells were also shown to play an important role in rapidly transforming FL. Rather than a numerical change, differences in spatial distribution and activation of CD4-positive cells were seen with predominance within the neoplastic follicles. This suggests a functional modulation of this cell population that may be growth supportive in this group of FL. This notion is supported by both in vitro and in vivo studies in mouse models. ${ }^{24,25}$ As also suggested by the combination of relatively increased expression of related genes, an increase in the relative contribution of T-helper 1 type T cells was observed. CD4+/CD25+ regulatory T cells, identified with FOXP3, are specifically involved in suppression of the active immune response. $^{26-28}$ In contrast to the prognostic role in solid cancers, it may be hypothesized in FL that this cell population may rather mediate the relative immunologically inactive state in good-prognosis FL. However, neither in terms of absolute cell numbers nor in spatial distribution (follicular, interfollicular, or diffuse) was our data able to support this idea.

As also indicated by our gene-expression profiling and analysis, dendritic cell populations may play a role in the growth properties and the clinical behavior of FL. ${ }^{29-31}$ Disruption of the CD21 immunohistochemical pattern, in the presence of intact CD23-positive meshworks within neoplastic follicles, may indicate functional heterogeneity and differential interactions with tumor cells in rapidly transforming FL. In contrast to the findings of others, we were not able to demonstrate a differential contribution of CD68-positive macrophages in relation to the risk of transformation or survival related to transformation. $^{23}$ The outline of this study was essentially different from ours, precluding complete comparison, however. A tissue microarray (TMA) platform was used, and overall and progressionfree survival were used as end points in contrast to transformation with essentially different criteria of identifying poor-risk patients. Moreover, all patients received highly intensified treatment instead of standard cyclophosphamide, doxorubicin, vincristine, and prednisone (CHOP) -like therapy.

Our findings further support a hypothetical two-disease model for FL. ${ }^{8}$ Such a model may also be supported from a genetic standpoint. In an elegant study by Höglund et al, ${ }^{32}$ the temporal pattern of the accumulation of genetic alterations in FL was descibed and it was shown that there may be two mutually exclusive pathways in early clonal evolution. One pathway is characterized by $-6 \mathrm{q}$ and $+1 \mathrm{q}$ as early changes, in the other pathway by duplication $\operatorname{der}(18)$ and $+7 /$ +8 , which can be recognized as relatively early changes. The specific genetic make-up of the tumor cells may determine the intrinsic survival capacity and proliferative drive of the tumor cells, as well as the overall genomic instability. Importantly, molecularly different FL may dictate a different microenvironment and by immunologic cross talk are in a different manner influenced by T cells and accessory cells. The interacting network of genomic and immunologic factors then defines the clinical behavior of the tumor. ${ }^{8}$

In conclusion, FL has now been recognized as a disease of functional B cells in which the clinical behavior is determined by properties of the malignant cells in functional cross talk with the immunologic regulatory network. It will be a challenge over the coming years to dissect the labyrinth of cellular and molecular information into a comprehensible and clinically useful model of interactions. Such insights will be of the utmost importance in order to develop intelligent treatment strategies targeted to specific biologic mechanisms responsible for the ultimately fatal course of the disease.

\section{AUTHORS' DISCLOSURES OF POTENTIAL CONFLICTS OF INTEREST}

The authors indicated no potential conflicts of interest.

\section{AUTHOR CONTRIBUTIONS}

Conception and design: Annuska M. Glas, Laurent Knoops, Marie José Kersten, Daphne de Jong

Provision of study materials or patients: Marie José Kersten, Robby E. Kibbelaar, J. Han J.M van Krieken, Joke W. Baars, John Raemaekers, Philip M. Kluin, Daphne de Jong

Collection and assembly of data: Annuska M. Glas, Laurent Knoops, Leonie Delahaye, Robby E. Kibbelaar, Daphne de Jong

Data analysis and interpretation: Annuska M. Glas, Laurent Knoops, Leonie Delahaye, Marie José Kersten, Lodewyk A. Wessels, Ryan van Laar, Philip M. Kluin, Laura J. van 't Veer, Daphne de Jong

Manuscript writing: Annuska M. Glas, Laurent Knoops, Marie José Kersten, Daphne de Jong

Final approval of manuscript: Annuska M. Glas, Laurent Knoops, Leonie Delahaye, Marie José Kersten, Robby E. Kibbelaar, Lodewyk A. Wessels, Ryan van Laar, J. Han J.M van Krieken, Joke W. Baars, John Raemaekers, Philip M. Kluin, Laura J. van 't Veer, Daphne de Jong 


\section{REFERENCES}

1. Horning SJ, Rosenberg SA: The natural history of initially untreated low-grade non-Hodgkin's lymphomas. N Engl J Med 311:1471-1475, 1984

2. Horning SJ: Natural history of and therapy for the indolent non-Hodgkin's lymphomas. Semin Oncol 20:75-88, 1993

3. Solal-Celigny $P$, Roy $P$, Colombat $P$, et al: Follicular lymphoma international prognostic index. Blood 104:1258-1265, 2004

4. Nathwani BN, Harris NL, Weisenburger D, et al: Pathology and genetics of tumours of haematooietic and lymphoid tissues. Jaffe ES, Harris NL, Stein $\mathrm{H}$, et al (eds). Lyon, France, IARC Press, 2001, pp 162-167

5. The Non-Hodgkin's Lymphoma Classification Project: A clinical evaluation of the International Lymphoma Study Group classification of nonHodgkin's lymphoma. Blood 89:3909-3918, 1997

6. Yunis JJ, Frizzera G, Oken MM, et al: Multiple recurrent genomic defects in follicular lymphoma: $\mathrm{A}$ possible model for cancer. N Engl J Med 316:79-84, 1987

7. Horsman DE, Connors JM, Pantzar T, et al: Analysis of secondary chromosomal alterations in 165 cases of follicular lymphoma with $t(14 ; 18)$. Genes Chromosomes Cancer 30:375-382, 2001

8. de Jong D: Molecular pathogenesis of follicular lymphoma: A cross talk of genetic and immunologic factors. J Clin Oncol 23:6358-6363, 2005

9. Glas AM, Kersten MJ, Delahaye LJMJ, et al: Gene expression profiling in follicular lymphoma to assess clinical aggressiveness and to guide the choice of treatment. Blood 105:301-307, 2005

10. Dave SS, Wright G, Tan B, et al: Prediction of survival in follicular lymphoma based on molecular features of tumor-infiltrating immune cells. N Engl J Med 351:2159-2169, 2004

11. Eray M, Postila V, Eeva J, et al: Follicular lymphoma cell lines, an in vitro model for antigenic selection and cytokine-mediated growth regulation of germinal centre b cells. Scand J Immunol 57:545555,2003

12. Bohen SP, Troyanskaya OG, Alter $\mathrm{O}$, et al: Variation in gene expression patterns in follicular lymphoma and the response to rituximab. Proc Nat Acad Sci U S A 100:1926-1930, 2003

13. van de Vijver MJ, He YD, van't Veer LJ, et al: A gene-expression signature as a predictor of survival in breast cancer. N Engl J Med 347:1999-2009, 2002

14. NKI/AvL Department of Pathology Microarray Projects. http://www.nki.nl/nkidep/pa/microarray

15. Central Microarray Facility. http://microarrays .nki.nl

16. Brazma A, Hingamp P, Quackenbush J, et al: Minimum information about a microarray experiment (MIAME): Toward standards for microarray data. Nat Genet 29:365-371, 2001

17. Liu H, Li J, Wong L: Use of extreme patient samples for outcome prediction from gene expression data. Bioinformatics 21:3377-3384, 2005

18. Hughes $T R$, Marton $M J$, Jones $A R$, et al: Functional discovery via a compendium of expression profiles. Cell 102:109-126, 2000

19. Sturn A, Quackenbush J, Trajanoski, Z.: Genesis: Cluster analysis of microarray data. Bioinformatics 18:207-208, 2002

20. Tusher VG, Tibshirani R, Chu G: Significance analysis of microarrays applied to the ionizing radiation response. Proc Natl Acad Sci U S A 98:51165121, 2001

21. Wessels LFA, Reinders MJT, Hart AAM, et al: A protocol for building and evaluating predictors of disease state based on microarray data. Bioinformatics 21:3755-3762, 2005

22. Bair E, Tibshirani R: Semi-supervised methods to predict patient survival from gene expression data. PLoS Biol 2:511-522, 2004

23. Farinha $P$, Masoudi $H$, Skinnider BF, et al: Analysis of multiple biomarkers shows that lymphoma-associated macrophage (LAM) content is an independent predictor of survival in follicular lymphoma (FL). Blood 106:2169-2174, 2005

24. Martin I, Bonnefoix T, Roucard C, et al: Role of autologous $\mathrm{CD} 4+\mathrm{T}$ cell clones in human $\mathrm{B}$ nonHodgkin's lymphoma: Aborted activation and G1 blockade induced by cell-cell contact. Eur J Immuno 29:3188-3195, 1999

25. Egle A, Harris AW, Bath ML, et al: VavP-Bcl2 transgenic mice develop follicular lymphoma preceded by germinal center hyperplasia. Blood 103 : 2276-2283, 2004

26. Schwartz $\mathrm{RH}$ : Natural regulatory $T$ cells and self-tolerance. Nat Immunol 6:327-330, 2005

27. von Boehmer $\mathrm{H}$.: Mechanisms of suppression by suppressor T cells. Nat Immunol 6:338-344, 2005

28. Suri-Payer E, Amar AZ, Thornton AM, et al: $\mathrm{CD} 4+\mathrm{CD} 25+\mathrm{T}$ cells inhibit both the induction and effector function of autoreactive $T$ cells and represent a unique lineage of immunoregulatory cells. J Immunol 160:1212-1218, 1998

29. Chang $\mathrm{KC}$, Huang $X$, Medeiros LJ, et al: Germinal centre-like versus undifferentiated stromal immunophenotypes in follicular lymphoma. J Pathol 201:404-412, 2003

30. Thomazy VA, Vega F, Medeiros LJ, et al: Phenotypic modulation of the stromal reticular network in normal and neoplastic lymph nodes: Tissue transglutaminase reveals coordinate regulation of multiple cell types. Am J Pathol 163:165-174, 2003

31. Shiozawa E, Yamochi-Onizuka T, Yamochi T, et al: Disappearance of CD21-positive follicular dendritic cells preceding the transformation of follicular lymphoma: Immunohistological study of the transformation using CD21, p53, Ki-67, and P-glycoprotein. Pathol Res Pract 199:293-302, 2003

32. Hoglund M, Sehn L, Connors JM, et al: Identification of cytogenetic subgroups and karyotypic pathways of clonal evolution in follicular lymphomas. Genes Chromosomes Cancer 39:195-204, 2004

\section{Acknowledgment}

We acknowledge Peter Joosten, MD, and Gustaaf van Imhoff, MD; the pathologists of the Boven IJ Hospital, Amsterdam, Pathological Laboratories Kennemerland, Pathological Laboratories Enschede, and Zuiderzee Hospital; Lelystad for providing samples and clinical data; and Huib Storm and Marije Booman, for helpful suggestions and stimulating discussions. 